labe vor der Operation sorgfältıg das Eccem geheilt, die Kranke 2 Tage liungern lassen und 8 Tage nach der Operation blos Milch und Fleischbrühe gegeben.

2) Mitte August $1882 \mathrm{kam}$ der Restaurateur Möller von Wolfsbehringen wegeu einer eingeklemntenl Leistenhernie zu mir. Die Enklemnnung bestand erst einen Tag, aber die Gesclıwulst bot durch ihre dunkel blaurothe Farbe, ihre grosse Sclımerzhaftigkeit bei dem argen Verfall des kräftigen Mannes eın selır bedenkliches Bild. Die Einrichtungsversuche des Herrn Collegen $M$. waren olne Erfolg und seln schmerzhaft gewesen. Nach Eröffnung des Bruchsackes fand sich nicht die Spur von Bruchwasser, der Bruchsack war prall ausgefüllt mit einer 28 Centimeter lingen Dünndarnıschlinge, welche mit den Bruchsack dicht verlöthet, ausgeschält wurde. Die Farbe des Darınes war dunkelblau; an elner nissfarbigen, glauzlosen Stelle ungefähr in der Mitte des vorgefallenen Darmrohrs ergoss sich aus einer kleinen dreieckigen Oeffnung ein breniger blutiger Inhalt. Zwei anwesende Herren Collegen sprachen sich entschieden gegen eine sofortige Anlegung einer Darmnaht aus, da eine solche nıcht gehalten hätte und die Grenze des vermuthlich eıne Darmuaht ertragenden Gewebes nicht zu erkennen war. Die Schinge

\section{Zwei Fälle von Darmfistel durch Bauchschnitt und Darmnaht geheilt.}

\author{
Von
}

\section{E. Meusel, Gotha.}

Am 20. December 1882 hat Herr Professor von Bergmann in der Sitzung der medicinischen Gesellschaft seine Erfahrungen über die Be$h$ andlung des Anus praeternaturalis mitgetheilt, und hat sich daran eine Discussiou geschlossen, welche mich veraulasst dem bereits vorliegenden Material 2 Fälle aus meiner Behandlung linzuzufügen.

1) Frau Ernstine Oschmann, 28 Jahre, aus Engelsbach bei Friedrichroda wurde mir am 28. Mai 1881 von Herrn Dr. Keil zugeschickt mit dem Bericht, sie leide schon seit 10 Tagen an einer Undurchgängigkeit des Darmrohres, seit 8 Tagen an Kothbrechen, Abführmittel und Eingiessungen seien olhne Erfolg gewesen, die Stelle des Verschlusses habe sich von vorneherein nicht feststellen lassen. Die Frau hatte einen enorm aufgetriebeuen Leib, gleichın̈ssig tynıpanıtischen Ton über den ganzen Bauch, nirgends eine bämpfung oder härtere Stelle. Der Puls war klein und rasch, die Temperatur niedrig, die Frau gleichgiltig gegen ihr Sclicksal.

Ich schritt sofort zun Bauchschnitt in der Linea alba, zwischeu Symphyse uud Nabel, erfasste eine stark anfgeblasene Dünndarmschlinge, legte einen Längsschuitt von ungefähr 2 Centımeter an, und vernältte die Ränder mit dem Peritoneum der Bauchwunde. Es entwichen Gase und sehr viel grüngefärbter Darminhalt, kothig riechend. Die Frau befaud sich bald wohler, nach 8 Tagen wurden die Nähte entferut. Schon uach 4 Tagen stellte sich Stuhlgang aus dem After ein, so dass die Frau bald nur Flüssigkeit aus der Fistel entleerte, wälırend fester Koth per anum ausgeschiedeu wurde. Alle Versuche die Fistel zum Verschluss zu bringen, Aetzungen, Cauterisationen mit denı galvanocaustischen Brenner blieben ohne Erfolg. Die junge Frau empfand das Leiden niclıt nur als ein sehr lästiges, sie fing auch au, schwächer zu werden und ging deshalb endlich im Frühling 1882 auf meinen Vorschlag ein, sich operiren zu lassen.

Bür die Anwendung der Dupuytren'schen Scheere lag keine Indication vor, da keiu Sporn bestand, welcher eine Ursache der Nichthein lung gewesen wäre. Auch fällt ein solcher Sporu von selbst weg, wenn man die Bauchhöhle öffnet und deu Darm bloslegt. Ich machte eiueu Bauchschuitt oberhalb der Fistel, dass ich bequem mit 2 Fingeru eingeheu und das mit dem Loch versehene Darmstück umgehen konnte. Ein besonderes Instrunıent, um das Darmrohr abzuschliessen, halte ich bei einiger Geschicklichkeit für unnöthig. Indem jch den Darnı anzog, kounte ich ohne Schwierigket die Fistel lostrenuen und den Darm inımer so halten, dass das freigewordene Stück ausserhalb des Bauches lag. Die Fistelränder wurden abgetragen, die Darmnalıt mit $\tilde{3}$ Catgutfäden angelegt, Versenkung des Darmrohrs, Schluss der Bauchwunde. Die fieberlose Heilung war in 10 Tagen beendet, einige Wochen wurde eine sorgfältige Diät beobachtet. Gegenwärtıg befindet sich die Frau wohl und ohne jede Beschwerde.

Sollte ich wieder in die Lage kommen, eiue solche Fistel anzulegen, so würde ıch nicht die Linea alba wählen, die jedenfalls wenig beiträgt, die Verhältnisse für den Verschluss günstig zu machen, wenigstens weist darauf die Entstehung von Hernien nach Laparotomien hin. Icl

1) Velpeau, l. c.

2) Dupuytren, Lecons orales de clin. chir. Tom. IV, Art. I.

3) Demme, Militär-chirurg. Studien, Würzburg 1863, I pag. 208.

$\left.{ }^{4}\right)$ Archiv für kliniscbe Chirurgie Bd. XI pag. 485

5) Arehiv für klinische Chirurgie Bd. XXI, pag. 218

6) Dieffenbach, Operative Chirurgie, 1845 Bd. I, pag. $720-722$ wurde also sorgfältig entleert, miıt warmer Sublimatlösung gewaschen, reponırt und das kleine Loch mit 3 Nähten in der Bruchpforte fixırt Trotzdem nach 14 Tagen Stuhlgang per anum erfolgte und bei der Kleinheit der Fistel eine spontane Heilung möglich schien, wurde eine solche nicht erzielt, eine Cauterisation machte die Sache nur schlechter. Der ungeduldige Patient drang auf eine Naht. November 82. Ith eröflnete die Bauclıhöhle ungefälır 2 Zoll oberhalb der Fistel, ging ınit den Fingern ein und hob den Darm von der Fistel ab, indem jch dıe Ränder lospräparirte. Es war nicht leicht das Peritoneum der Bauchwand vou dem Ueberzug des Darmes bei der breiten Verwaclsuug zu scheiden. Die Darmspalte war grösser und länger als ich nach dem ursprünglichen brandıgen Eınrıss und nach der äusserlichen Kleinheit vermuthet hatte. Indessen folgte die Richtuug ziemhch genau der Längsachse und war die Breıte des Defectes so gering, dass die Naht das Lumen micht wesentlich verringerte Ich legte 6 Catgutfäden an, versenkte deu Darm und schloss die Bauclwunde. Hellung nach 10 Tagen. Am 14. Tage fuh Patient nach Eisenach und ging wieder seinen Geschäften nach.

V. R. Thoma (Heidelberg), Untersuchungen über die Grösse und das Gewicht der anatomischen Bestandtheile des menschlichen Körpers im gesunden und kranken Zustaude. Leipzig. F. C. W. Vogel. 1882. VI. 285 S. $8^{0}$. Preis 8 M. Referent Karl Bardeleben.

Bekanntlichı unterliegen Grösse und Gewichtı des menschlıcheu Körpers sowie seiner Theile (Organe) recht erheblichen individuellen Sclıwankungen, wie dies ja duch auf auderen Gebieten der Naturwissenschaft beobachtet wird. Trotzdem weisen die anatomischen Thatsachen darauf hin, dass für jede Art lebender Wesen ein allgemeiner Bauplan existirt oder aufgestellt werden kann, nach welchem sich das einzelue Individuum annäherungsweise riclitet, wenn auch die iudividuellen Abweichungeu oft erhebliche zu sein scheinen, sodass nıan es auf deu ersten Blıck für unmöglich hält, in das Gewirr und den Wechsel der Einzellälle Ordnung und Gesetz hineinzubringeu. Hiermit soll jedoch keine "Constanz der Arten" in Allgemeinen behauptet werden, im Gegenthell. Die Zeiträume, über die wir bei anatonischen und anthropologischeu Untersuchungen verfügen, sind inı Verhältniss zı dem enormen Alter unserer Species viel zu geringfügig, als dass wır uicht für unser Jahrhundert, wohl auch Jahrtausend eine Norm oder den Bauplan aufstellen könnten. So etwa der Gedankengang des Verfassers, wie er sich im Vorworte darstellt. Auf Raceuverschiedenheiten nimmt derselbe keine Rücksicht; das Material bezieht sich nur auf Deutschland, speciell die Heidelberger Gegend, eine Thatsache, die hervorgehoben werden soll, da Ref. davon überzeugt ist, dass auch innerlaalb Deutschlands, abgeselsen von der Schädelbildung, sich melırere anatomiscle Typen unterscheiden lassen.

Die erste Aufgabe, so fährt Verf. fort, wird es nun seiu, zu prüfen, ob eln allgerneiner Bauplan für den Menschen wirklıch existirt, zwettens ihn dann in seinen Einzelheiten nach Maass und Gewicht festzustellen. Bekanutlıch hat Quetelet gezeigt, dass die individuelleu Abweıchungen der Körperlänge und des Körpergewichts von der ideellen Norm bezüglich ihrer Häufigkeit und ihrer Grösse (Stärke) sich in einen verhältnissmässig einfacheu matlematischen Ausdruck bringen lassen. Verf. hat sich nun die Aufgabe gestellt, diese Erfahrungen Quetelet's weiter zu verfolgen und zu untersuchen, ob auch die anatomisclieu Bestandthelle, die Organe und die Gewebselemente (z. B. Blutzellen) die gleiche Regelmässigkeit erkenneu lassen, wie Körperlänge und Körpergewicht. Herbei hat sich nun eine allgemeine Theorie der individuellen Verschiedenheiten des menschlichen Körpers ergeben, deren Bedeutung nicht hoclı genug angescllageu werden kann, da sie die Methoden und Mıttel zu der Lösung einer Reihe interessanter ana- 
tomischer Fragen, sowie zu einer scharfen und objectiven Kritik der Ergebnisse der zahlreichen Untersucliungen auf dem weiten Felde der normalen und pathologischen Anatomie darbietet.

Ref. giebt hier aus Gründell, die in der Natur der Sache liegen, weder eine Recension noch ein Referat über das Buch von Thoma, sondern nur ein kurzes Inhaltsverzeichnıss, indem er allen Interessenten die Lectüre des Orıginals dringend empfiehlt. Verf. hat im ersten Theile die theoretischen Betrachtungen, in zweiten die Beobachtungsreihen vereinigt, eine jedenfalls practische Gruppirung. Die sieben Capitel des ersten Theiles enthalten Folgendes: 1) die indıviduellen Verschiedenheiten; 2) die Norm und die individuellen Abweichungen; 3) die Bestimmung der Norm und des walıscheinlichen Werthes der individuellen Abweichungen aus gegebenen Beobachtungen; 4) die relativen Maasse und Gewıchte; 5) die pathologisclıen Veränderungen der Grösse und des Gewichtes der Organe; 6) die Beobachtungsfehler; 7) Allgemeine Technik der Beobachtung. - Der zweite Theil bringt Beobachtungsreihen über: 1) Körperlänge und Körpergewicht; 2) das Gewicht des Herzmuskels und des Herzens; 3) das Gewicht der Nieren; 4) der Durchmesser der grossen Blutgefässe; 5) die Messung und die Zälllung der Zellen des Blutes. Die Beobachtungen sind theils eigene, theils fremde. - In einem Anhang werden niathentatische Erörterungen gegehen für die Theorie der individuellen Verschiedenheiten im allgemeinen, sowie ihre Grösse und Häufigkeit im speciellen, für den wahrscheinlichen Werth der individuellen Abweichungen der Summe des Gewichts oder der Grösse zweier Organe, für die relatıven Grössen und die relativen Gewichte der anatomischen Körperbestandtheile u. s. f. Das zwölf Seiten umfassende Litteraturverzeichniss ist, weln auch nicht ganz, so doch sehr vollständig.

Abgeselıen von dem neuen Material (Nieren, Blutgefässe, Blutzellen) hat Thoma sonach eine sehr erwünschte theoretische Grundlage für anatomische Untersuchungen von Grösse und Gewicht der Organe geliefert, eine Grundlage, die jetzt doppelt und dreffach werthroll wird, je mehr normale und pathologische Anatomen siclı diesem vor Kurzem noch fast ganz unfruchtbar erscheinenden Felde wissenschaftlicher Thätigkeit zuzuwendell beginnen. Der Fortschritt, welchen wir jetzt von dem frülıer üblichen „Durchschnitt" vermittelst des Individualisirens und der theoretisclı-matlıematischen Erörterung zur wirklichen „Norm“ mit Berücksichtigung der nothwendigen indıviduellen Abweichungen machen, ist ein noch unberechenbarer und in seinen Folgen für die gesammte Medicin unabselıbarer.

Vl. Birch-Hirschfeld. Lehrbuch der patlologischen Anato mie. 2. völlig unigearbeitete Auflage. Erster Band, allgem. Theil. 254 pp. mit 118 Abbildungen im Text. Leipzig, Vogel. Referent: Marchand.

Das Lehrbuch Birch-Hirschfeld's liegt uns in seinem ersten allgemeinen Theile in völlig neuem Gewande vor, und, wie wir gleich hinzufügen können, in mehr als einer Beziehung vervollkommnet. Das Bestreben des Verf., dem Buche eine weniger voluminöse Form zu geben, kann demselben gewiss nur zum Vortleile gereichen, namentlich mit Rücksiclt auf die Verbreitung unter den Studirenden. Der ganzen Anlage nach sclıent uns das Werk zwar auch jetzt noch mehr für den practischen Arzt, wenigstens nicht für den ersten Unterriclıt in der pathologischen Anatomie geeignet zu sein, denn leider haben ja unsere jungen Mediciner nur wenig Zeit zum Lesen, noch weniger zum Verarbeiten des Gelesenen und Gelörten. Der aligemeine Theil des Buches umfasst noch ziemlich dieselbe Settenzahl wie der gleiche Abschnitt der ersten Auflage, und zwar bei erheblich grösserem Format, so dass der lnhalt thatsächlich eine nicht unbedeutende Vermehrung erfalıren hat. Diese war gerechtfertigt, ja nothwendig geworden durch die grossen Fortschritte, welche in verschiedenen Gebieten der allgemeinen Pathologie und besonders der allgemeinen Aetiologı zu verzeichnen sind, und wir finden diese, beispielsweise die parasitären Krankheitsursachen, in eingehendster Weise berücksichtigt.

Die speciellen Theile des Werkes sollen dagegen in knapperer Form dargestellt werden.

Als eine wesentliche Verbesserung müssen wir es bezeichnen, dass an Stelle der wenig brauclibaren Tafeln der ersten Auflage eine grosse Zalıl von grösstentheils selır guten Abbildungen nach Originalpräparaten im Texte getreten ist. Bei manchen derselben würden wır etwas präcisere, wenn auch mehr schematische Conturen wünschen.

Zweckmässig sind ferner die Literaturnachweise an der Spitze der einzelnen Capitel.

Auf den lnhalt des Werkes einzugehen, behilten wir uns bis zum Erscheinen der übrigen Theile vor.
VII. Dr. Renk, Docent an der Universität, l. Assistent am hygien. Institute zu München: Die Kanalgase, deren lıygienische Bedeutung und technische Behandlung. München 1882. M. Rieger's Universitätsbuchhandlung. Referent: F. Beetz.

Die Wahrnehmung, dass bei der Anlage von Hausentwässerungen, Abortanlagen etc. aus dem Grunde die gröbsten Feller vorkommen, dass den ausführenden Handwerkern, welchen die Kenntniss der physikalischen Vorgänge in den Rohrleitungen fehlt, zu viel des Details überlassen zu werden pflegt, hat den Verf. zur Veröffentlıchung seiner Beobachtungen über das angeführte Thema veranlasst. Die Literatur, sowoll in hygienisch en wie in technischen Publicationen ist in der That eine sehr spärliche, und der Werth einer genauen Kenntniss der in Betracht kommenden Factoren leuchtet von selbst ein, wenn man erwägt, dass verfellte Anlagen der aus den Kanälen res. Abortgruben stammenden Emanationen nicht nur kein Hinderniss entgegenstellen, sondern denselben formlıch den Weg in die Wohnstätten wersen.

Das lnteresse, welches derartige Arbeiten für den Techniker haben, ist ja zweifellos; aber auch der Arzt - nicht nur der Theoretiker, sondern grade der practische Arzt - wird sich einer Keuntnissnahme der von Dr. Renk besprochenen Einrichtungen kaum entschlagen können. Mit Recht betonte von Pettenkofer, als er im Jahre 1863 auf die Einführung der Hygiene als obligaten Lehrgegenstand drang, dass auf jeden Hauseigenthüıner, an den von Salubritäts wegen irgend eine Anforderung gestellt werde, die Ansicht seines Hausarztes einen entscheidendel Einfluss habe, ob er darın etwas Nothwendiges und Nützliches, oder nur eine Polizeiquälerei erblicke.

Einen speciellen Einfluss auf die Verbreitung von Infectionsstoffen, epidemischen Krankheiten, oder auf eine besondere Disposition des 0rganismus für diese erkennt Verf. den Kanalgasen nicht zu, und bespriclit die lierüber geführten Controversen (Buchner, Wernich, Gruber) in ausfuhrlicher Weise.

Trotzdem müssen die gasförmigen Emanationen der Kanäle etc. von unseren Wohnungen ferngehalten werden, und nicht blos aus ästhetischen Gründen, sondern weil der Körper auf das beständige Einathmen verdorbener Luft in ähnlicher Weise reagirt, als wenn demselben längere Zeit hindurch die Genussmittel der Nahrung vorenthalten worden wären.

Verf. bespricht in den einzelnen Kapiteln: die Kanalluft, deren hygienische Bedeutung, die Ursachen des Eindringens der Kanalluft in die Häuser und dıe Vorrichtung zur Verhütung desselben, sowie die Ventilation der Kanäle.

Mehr als einmal ist man bei der Lectüre des Buclies überrascht, zu sehen, wie Vieles in unserer Wohnungsliygiene in Argen liegt, das leicht zu bessern wäre, und man fühlt sich an den in von Pettenk ofer's Vorträgen über Canalisation erwähnten Hauseigenthümer erinnert, der die im Interesse der Miether gewünsclıte Abstellung von Missständen ablehnte, weil er „sein Häusel sclımecken" wolle.

Hoffen wir, dass das Buch zur Verbreitung gesunder Ansichten, und zur Einführung besserer Einrichtungen beihelfend, einen entsprechend grossell Leserkreis finden möge. 